Summary of 1994-5 Activities Grant FG03-88ER-45375

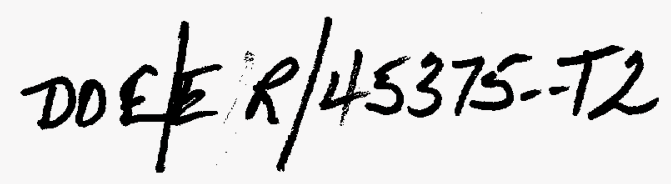

\title{
Confinement of Block Copolymers
}

Thin films of diblock copolymers self-assemble into a multilayered structure due to the preferential interactions of the blocks with the air surface and the substrate interface. This multilayering forces the film thickness at any point to be given in terms of an integral or half integral multiple of the equilibrium period. If the initial film thickness is not commensurate with this constraint, then islands or holes, with a step height of one period, form on the surface.

In these studies the surface topography was prohibited by confining the diblock copolymer between two hard walls. A novel technique was developed to ensure proper confinement. It was found that as the film thickness of the confined copolymer increased, the copolymer still self-assembled into a multilayered structure, but the period of the copolymer changed to compensate for the constraint. A cyclic contraction and expansion of the period was observed wherein the amount of deformation to the copolymer depended upon the number of confined layers. In particular, the expansion or contraction decreased as $1 / n$ where $n$ were the number of layers. In all cases, an integral number of layers was formed between the two confining surfaces. However, with increasing film thickness, a first order phase transition was observed in the number of confined layers. In addition, the interfacial width between the copolymer microdomains was reduced when the copolymer period was less than the equilibrium value and increased for expanded periods. This effect is due to the suppression and enhancement of the capillary waves between adjacent layers.

\section{Free Surface Confinement}

For diblock copolymers which are in the anti-symmetric absorption condition, i.e. one block preferentially located at the air surface and the other block at the substrate interface, the film thickness, after the formation of the multilayered structure, is given by $(n+1 / 2) L_{0}$, where $L_{0}$ is the equilibrium period. If the initial film thickness is not too different from this value, it is found that the formation of a surface topography can be suppressed for extended periods of time. In fact, the formation of islands, while suppressed, forms after extended annealing, whereas the formation of holes is retarded. This effect results from the activation barrier that must be crossed to form the surface topography and is due to critical fluctuations at the interface and the activation energy that must be overcome to form the topography.

\section{Effects of Substrate Interactions}

By using a thin layer of a random copolymer at the two interfaces to confine a diblock copolymer, the effective interaction energy of the block copolymer with the interfaces can be varied. Random copolymers are particularly suited for this purpose since the composition of the copolymer can be varied in a straightforward manner in the synthesis. In the case of PS and PMMA, using a random copolymer with a 0.68 fraction of PS in the random copolymer, the interactions of the PS and PMMA blocks with the interface are balanced. Using this random copolymer as the confining interface, it was found that the orientation of the lamellar microdomains with respect to the surface could be varied by simply changing the film thickness. For layer thicknesses close to $(n+1 / 2) \mathrm{L}_{0}$, a thickness of maximal frustration, strong density correlations in the plane of the film were evident from neutron reflectivity studies. These results were confirmed by independent transmission

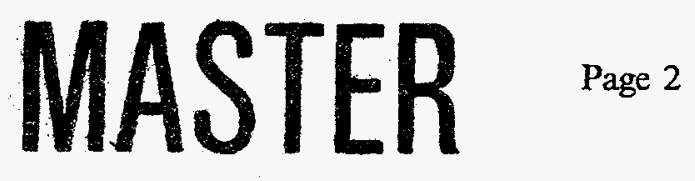




\section{DISCLAIMER}

Portions of this document may be illegible in electronic image products. Images are produced from the best available original document. 
electron microscopy studies. Slight changes in the film thickness resulted in an orientation of the lamellar microdomains parallel to the interface. These results confirm our previous theoretical arguments on the manner in which the microdomain orientation could be altered and represent a significant step towards controlling the morphology on a nanoscopic level.

\section{Random Copolymers at Homopolymer Interfaces}

Random copolymers placed at the interface between two homopolymers are an effective means by which the interfacial toughness can be improved. Studies on the toughness between PS and PMMA using random copolymers clearly show that maximal strength is not obtained at a styrene fraction of 0.5 but rather at 0.68 . This asymmetry in the concentration represents a significant departure from other experimental results but can be traced to a concentration dependent segmental interaction parameter. Solubility studies show that a random copolymer with this composition is insoluble in both PS and PMMA. Neutron reflectivity studies also showed that the interfacial broadening was symmetric when the 0.68 copolymer was used. Therefore, the effectiveness of a random copolymer depends critically upon the insolubility of the random copolymer with the two homopolymers.

\section{Phase Separation in Thin Film Polymer Mixtures}

The phase separation in thin film mixtures of two homopolymers was investigated. The temperature and mode of phase separation was found to depend strongly upon the interactions of each homopolymer with the air and substrate interfaces and with each other. Generally, the temperature at which phase separation occurred was elevated due to the preferential interactions of the homopolymers with the interface. This produced an excess of each component at either one or both interfaces which effectively extracts homopolymer from the bulk of the film. This shifts the phase diagram due to the compositional changes and retards a lateral phase separation of the homopolymers. When phase separation occurs, a marked roughness in the roughness of the air interface is found which can, depending upon the surface tensions of the components and the interfacial tension, lead to a dewetting phenomenon.

\section{Buffing of Polymer Surfaces}

A long outstanding question in liquid crystal display technology is the origin of the orientation of the liquid crystal when placed in contact with a polymer that has been gently rubbed with a velour cloth under minimal loads. Considering that the polymer used is an aromatic polyimide, with a glass transition temperature in excess of $350^{\circ} \mathrm{C}$, and the buffing is done at room temperature, it is remarkable that buffing does anything to the polyimide surface. Using grazing incidence x-ray scattering, GIXS, it was shown that the buffing produces a substantial orientation of the polymer chains in the vicinity of the surface, while the bulk of the material is unaffected by the buffing process. Consequently, the orientated chains on the surface behave as a molecular template for the liquid crystals and give rise to the observed behavior. Other mechanisms for the orientation of the liquid crystals, as for example an electrostatic charge on the surface or the grooves placed on the surface during buffing, could be excluded as the primary mechanism of alignment. It should be noted that the amount of deformation of the chains at the surface was far in excess of any orientation that could be obtained with a bulk sample during cold drawing.

\section{Near Edge X-ray Absorption Fine Structure Spectroscopy}


Near edge x-ray absorption fine structure, NEXAFS, spectroscopy was used to examine the orientation in buffed polyimide. Using Auger electrons ejected from the sample or the total number of electrons ejected from the surface, coupled with the polarization 0 of the incidence $x$-ray beam at a synchrotron source, permitted the evaluation of the orientation function of the polymers over a depth of $10 \AA$ and $100 \AA$, respectively. The total electron yield NEXAFS near the carbon and oxygen edges, were in full agreement with the GIXS results. However, the Auger results showed that, at the surface, the orientation of the polyimide molecules was much greater. Studies performed as a function of the load, distance and speed of buffing were, also, performed. For loads in excess of $1.6 \mathrm{~g} / \mathrm{cm}^{2}$ and buffing distances in excess of $50 \mathrm{~cm}$, the amount of orientation was saturated. However, no dependence on the speed of buffing was found. Thus, it is the effective strain of the molecules on the surface that is most critical in producing the alignment of chains. 


\section{Publications}

1. Near Surface Alignment of Polymers in Rubbed Films, M.F.Toney, T.P.Russell, H.Kikuchi, J.A.Logan, J.M.Sands and S.K.Kumar, Nature, 374, 709(1995).

2. Thermal Expansion of Thin Diblock Copolymer Films, P.Bassereau and T.P.Russell, Israel J. Chem., 35, 13 (1995).

3. Two Dimensional Ostwald Ripening in Symmetric Diblock Copolymer Films: A Reply, T.P.Russell, P.Bassereau, D.Brodbeck, H.R.Brown and K.R.Shull, Phys. Rev. Lett., 74, 4960 (1995).

4. Adsorption Studies of Symmetric Diblock Copolymers on a Solid Surface by Evanescent Wave Ellipsometry, M.W.Kim, T.Moses, W.Chen, Y.R.Shen and T.P.Russell, Macromolecules, 27, 7490 (1994).

5. Solvent Penetration into Ordered Thin Films of Diblock copolymers, H.Lin, A.Steyerl, T.P.Russell, S.K.Satija and A.Karim, Macromolecules, 28, 1470 (1995).

6. Small Angle Neutron Scattering Studies on Ultrathin Polymer Films, T.P.Russell, P.Lambooy, J.G.Braker, P.Gallagher, S.K.Satija, G.J.Kellogg and A.M.Mayes, Macromolecules, 28, 787 (1995).

7. A Method to Confine Thin Solid Organic Films Between Rigid Flat Plates, P.Lambooy, J.R.Salem and T.P.Russell, Thin Sol. Films, 252, 75 (1994).

8. Rheology of the Lower Critical Ordering Transition, T.E.Karis, T.P.Russell, Y.Gallot and A.M.Mayes, Macromolecules, 28, 1129 (1995).

9. Surface Orientation of Liquid Crystalline Poly(alkylsilanes), B.J.Factor, T.P.Russell and M.F.Toney, Acta Polym., 46, 60 (1995).

10. Observed Frustration in Confined Block Copolymers, P.Lambooy, T.P.Russell, G.J.Kellogg, A.M.Mayes, P.D.Gallagher and S.K.Satija, Phys. Rev. Lett., 72, 2899 (1994).

11. Block Copolymer Mixtures as Revealed by Neutron Reflectivity, A.M.Mayes, T.P.Russell, V.R.Deline, S.K.Satija and C.F.Majkrzak, Macromolecules, 27, 7447 (1994).

12. The Near Surface Structure of Aromatic Polyimides: The Effect of Precursor Isomers, B.J.Factor, T.P.Russell and M.F.Toney, Faraday Discuss., 98, 319 (1994). 


\section{Research Directions}

\section{Controlling Microdomain Orientation}

A key element in the utilization of thin films of diblock copolymers in any application is being able to force the copolymer to orient in a specific direction. Three different routes will be pursued to this end. These are:

Controlling surface interactions

Using electric fields

Using heterogeneous substrates

In an extension of the work completed during the previous year, random copolymers have been synthesized in which there is an endgroup placed on the copolymer that can covalently react with the substrate. This provide a mechanism by which the surface potential can be controlled without the possibility of the copolymer diffusing into the confined diblock copolymer film. Studies will be performed, initially, on films with a free surface to gain a precise understanding of the coupling between the random copolymers and the diblock copolymer. Providing the theoretical considerations are correct, then by combining the film thickness and the variable surface interactions, a precise delineation of the conditions under which parallel and vertical orientation of the lamellar microdomains with respect to the surface will determined.

For bulk specimens, electric field alignment of diblock copolymers is quite inefficient. Shear induced alignment, where mechanical energy is coupled into the copolymer, is much more efficient. However, for thin films this should not be the case. With thin films, using low voltages, the effective field can be quite large. Consequently, electric field alignment should be quite pronounced in the thin film geometry. In situ reflectivity studies will be performed under an electric field to follow the rate at which copolymer alignment will occur. These studies will be augmented by transmission electron microscopy studies to quantify the resultant morphology. Finally, the variation in the interaction strength of the substrate using the anchored random copolymers. will be coupled into the electric field alignment studies.

\section{Heterogeneous Substrates}

Recently, a novel means of producing a heterogeneous surface was developed. To date, surfaces with $300 \AA$ lines of one material, as for example $\mathrm{Au}$, can be produced on an $\mathrm{SiO}_{2}$ substrate have been produced. However, the method of producing the lines on the surface must be optimized and the chemical composition of the surface must be varied to produce the most desirable substrate.

Having a heterogeneous substrate that is smooth on the $\AA$ size scale is essential for many different problems. In particular, the behavior of a polymer mixture or a diblock copolymer on a laterally heterogeneous surface are unexplored experimentally. In the case of mixtures, studies will be performed whereby the size scale of the lateral phase separation will be investigated as a function of film thickness and size scale of the surface features. In the case of diblock copolymers, studies will be performed to determine the commensurability of the substrate with the equilibrium diblock morphology in order to optimize the control over the orientation of the morphology.

Surface Alignment of Polymers 
The studies begun on the mechanical orientation of homopolymers at a surface will be extended during the upcoming period. In particular, both GIXS and NEXAFS studies will be performed on different polymers where the amount and type of ordering in the polymer will be varied. These studies should lead to a direct understanding of relaxation mechanism at polymer surfaces and the conformation of polymer molecules at surface.

\section{Random Copolymers at Dissimilar Homopolymer Interfaces}

Studies will be performed using $\mathrm{AB}$ random copolymers at the interface between $\mathrm{AC}, \mathrm{BD}$ and $\mathrm{CD}$ homopolymer interfaces. The insight gained on previous studies have clearly pointed to a dissolution mechanism of the random copolymer into the adjacent homopolymer layers as giving rise to a weakening of the interfacial strength. It is necessary, however, to push this idea further by varying the interactions of the random copolymer with either or both homopolymer layers. This will be both favorable and unfavorable interactions.

\section{Thin Film Neutron scattering}

Previously we showed that is was possible to obtain neutron scattering results on ultrathin diblock copolymer films and on polymer mixtures. Studies are currently in process to determine the configuration of of isolated polymer chains in thin films. Studies will, also, be performed to determine the chain configuration of polymer molecules at the interface between two homopolymers and in confined geometries.

\section{Pressure Dependence of Copolymer Ordering}

Recently we discovered a diblock copolymer that underwent a transition from the disordered state to the ordered state upon heating. This unusual behavior can be explained by equation of state arguments which depend strongly upon the thermal expansion and compressibility of the polymer. Here, a series of neutron and x-ray scattering studies will be performed on this copolymer as a function of applied hydrostatic pressure. The application of pressure should, if the arguments are correct, lead to an elevation of the ordering temperature and to a quantitative understanding of this rather unusual behavior.

\section{Phase Separation of Confined Mixtures}

The studies done up to now on the phase separation of thin films of polymer mixtures have relied on the film thickness to provide the constraint. This, however, is not the most ideal manner in which these studies should be performed, since there is a free surface present. In these studies, the confinement used for diblock copolymers will be used to investigate the phase separation of mixtures confined between two hard walls. This removes the possibility of the formation of rough surfaces and potential dewetting behavior. Such studies are ideally suited for comparison with theoretical arguments on the subject.

\section{DISCLAIMER}

\footnotetext{
This report was prepared as an account of work sponsored by an agency of the United States Government. Neither the United States Government nor any agency thereof, nor any of their employees, makes any warranty, express or implied, or assumes any legal liability or responsibility for the accuracy, completeness, or usefulness of any information, apparatus, product, or process disclosed, or represents that its use would not infringe privately owned rights. Reference herein to any specific commercial product, process, or service by trade name, trademark, manufacturer, or otherwise does not necessarily constitute or imply its endorsement, recommendation, or favoring by the United States Government or any agency thereof. The views and opinions of authors expressed herein do not necessarily state or reflect those of the United States Government or any agency thereof.
} 